\title{
Contribution of Diabetes to the Incidence and Prevalence of Comorbid Conditions (Cancer, Periodontal Disease, Fracture, Impaired Cognitive Function, and Depression): A Systematic Review of Epidemiological Studies in Japanese Populations
}

\author{
Hirokazu Tanaka ${ }^{1,2, *}$, Noriko Ihana-Sugiyama ${ }^{1,3, *}$, Takehiro Sugiyama ${ }^{1,2}$, and Mitsuru Ohsugi1 ${ }^{1,3}$ \\ ${ }^{1}$ Diabetes and Metabolism Information Center, Research Institute, National Center for Global Health and Medicine, Tokyo, Japan \\ ${ }^{2}$ Department of Public Health, Graduate School of Medicine, the University of Tokyo, Tokyo, Japan \\ ${ }^{3}$ Department of Diabetes, Endocrinology and Metabolism, National Center for Global Health and Medicine Center Hospital, Tokyo, Japan \\ Received July 7, 2017; accepted November 27, 2017; released online June 23, 2018
}

\begin{abstract}
Background: Several epidemiological studies have determined the relationship between diabetes and the incidence and/or prevalence of recently identified comorbid conditions (cancer, periodontal disease, fracture, cognitive impairment, and depression). These relationships may vary by country or race/ethnicity. We aimed to systematically review studies in this field conducted with the Japanese population because such a review in the Japanese population has never been undertaken.

Methods: We conducted systematic literature searches in PubMed and Ichushi-Web databases for studies published until December 2016. Studies comparing the incidence and/or prevalence of the comorbidities among the Japanese population were included. The studies were classified as integrated analyses, cohort studies, case-control studies, or cross-sectional studies.

Results: We identified 33 studies (cancer: 17, periodontal disease: 5, fracture: 5, cognitive impairment: 4, and depression: 2). Although several cohort studies and meta-analyses had assessed the development of cancer in diabetes, there was scant epidemiological evidence for the other conditions. Indeed, only one cohort study each had been conducted for periodontal disease, fracture, and cognitive impairment, whereas other evidence was cross-sectional, some of which was induced from baseline characteristic tables of studies designed for other purposes.

Conclusion: In Japan, there is insufficient evidence about the relationship between diabetes and the incidence/prevalence of periodontal disease, fracture, cognitive impairment, and depression. By contrast, several cohort studies and integrated analyses have been conducted for the relationship with cancer. Further studies should be undertaken to estimate the contribution of diabetes on the incidence/prevalence of comorbidities that may be specific to the Japanese population.
\end{abstract}

Key words: diabetes mellitus; diabetes complications; epidemiological studies; review; Japan

Copyright $\odot 2018$ Hirokazu Tanaka et al. This is an open access article distributed under the terms of Creative Commons Attribution License, which permits unrestricted use, distribution, and reproduction in any medium, provided the original author and source are credited.

\section{INTRODUCTION}

Diabetes mellitus has become increasingly common, and it is known that inadequate disease control can undermine quality of life through diabetes-specific symptoms ${ }^{1}$ and conventional diabetic microvascular complications. ${ }^{2}$ In addition, macrovascular complications, such as coronary heart disease and stroke, occur more frequently in patients with diabetes and are the leading causes of death for those with diabetes in many countries. ${ }^{3}$ With their increased prevalence, diabetes and its associated complications are now regarded as a global burden. ${ }^{3}$ Thus, reducing the prevalence and economic burden of diabetes has become an important goal of medical care and health policy., ${ }^{4,5}$

Recently, comorbidities, such as cancers, fractures, and dementia, have been viewed as diabetes-related and can impair both the quality of life and the survival of patients. ${ }^{2}$ Given that the life expectancy of patients with diabetes has been prolonged because of improved care for conventional major diabetic complications, ${ }^{6}$ these newly recognized comorbidities and the increase in the number of elderly patients with diabetes have emerged as new healthcare challenges. Comparing the incidence and prevalence of these conditions between individuals with diabetes and those without is required to assess whether diabetes prevention or its management helps prevent these conditions.

Some studies have attempted to reveal the relationship between diabetes and these emerging comorbidities in the Japanese population. However, we suspect that these newly identified comorbidities are poorly captured in epidemiological studies and that their burden is large in Japan; the number of patients with

Address for correspondence. Mitsuru Ohsugi, Diabetes and Metabolism Information Center, Diabetes Research Center, Research Institute, National Center for Global Health and Medicine, 1-21-1 Toyama, Shinjuku-ku, Tokyo 162-8655, Japan (e-mail: mosugi-tky@umin.net).

*These authors equally contributed to this work. 
diabetes was estimated at 7.2 million adults in $2015 .{ }^{4}$ In addition, we considered that the most of evidence that was shown in the Japanese guideline for diabetes care was produced in other countries. ${ }^{7}$ Crucially, the relationship between diabetes and these newly identified diabetes-related conditions may vary by country or ethnicity. Within this context, in this systematic review, we aimed to summarize the current research about the incidence and prevalence of the newly recognized diabetes-related conditions among patients in Japan.

\section{Scope}

Among the conditions that appear related to diabetes, we focused on five: cancer, periodontal disease, fracture, impaired cognitive function/dementia, and depression. These were selected for the following reasons: (1) the pathophysiological relationships between diabetes and the complications are well established; (2) epidemiological evidence has been reported linking diabetes and those conditions, at least outside of Japan; (3) the burdens of those comorbidities on quality of life, complexity of medical care, and/or longevity of patients are significant; (4) preventing or treating diabetes has been shown to improve outcomes in those complications, or there are remedies for treatment or alleviation of symptoms; and (5) the potential impact of revealing the incidence and prevalence of those conditions is considerable in the areas of epidemiology, medical economics, and diabetes management. The American Diabetes Association guideline referred to six other comorbidities (autoimmune diseases, fatty liver disease, hearing impairment, human immunodeficiency virus, low testosterone in men, and obstructive sleep apnea). However, we excluded these comorbid conditions because they did not meet the criteria above. For instance, we excluded fatty liver disease due to the following reasons: it results from multiple factors, including patients' lifestyle, and not solely from diabetes; it does not directly influence the quality of life; and it does not require specific medical care, unless it develops into cirrhosis or liver cancer. Although the scope of our research was limited to the Japanese population, we first summarize the pathophysiological basis for including the selected complications, together with the epidemiological data.

\section{Cancer}

There is a growing body of evidence that diabetes is associated with an increased incidence of cancer, which is supported by a consensus report from the American Diabetes Association and the American Cancer Society in 2010. ${ }^{8}$ Many mechanisms have been proposed to explain the link between increased cancer risk and diabetes, including insulin resistance and hyperinsulinemia, increased oxidative stress caused by hyperglycemia, chronic inflammation, and abnormalities in the levels of adipocytokines. ${ }^{9}$ However, epidemiological evidence is not straightforward to interpret because risk factors (eg, age, sex, obesity, and inappropriate diets) may confound the relationship. ${ }^{9}$ An umbrella review of 27 meta-analyses looking at the relationship between type 2 diabetes and cancer incidence or mortality was published in $2015 .{ }^{10}$ All of the original 27 meta-analyses reported increased risks of developing cancer among those with diabetes; however, the review found that less of the cancer incidence could be significantly associated with type 2 diabetes after considering the substantial heterogeneity, small study effects, and excess significance in the studies. ${ }^{10} \mathrm{We}$ focused on the development of cancer in patients with diabetes in Japan.

\section{Periodontal disease}

Periodontitis refers to chronic inflammation of the gingiva, which leads to periodontal pocket formation, loss of connective tissue attachment, and alveolar bone resorption. There are several good-quality studies showing the relationship between diabetes and periodontal disease; for example, in a large-scale cohort study of Pima Indians in the United States, the rate of periodontal disease in those with type 2 diabetes was 2.6 times higher than in those who had no periodontal disease. ${ }^{11}$ There was another useful analysis by the National Health and Nutrition Examination Survey III in the United States. This cross-sectional populationrepresentative survey showed that, compared to patients without diabetes, those with poorly controlled (HbA1c >9.0\%) and moderately controlled (HbA1c $\leq 9.0 \%$ ) type 2 diabetes had 2.9fold and 1.6-fold higher rates of periodontitis, respectively. ${ }^{12}$ The causal relationship between diabetes and periodontitis is considered bidirectional, with periodontitis being associated with whole-body insulin resistance ${ }^{13}$ and diabetes and poor glycemic control being known risk factors for development and progression of periodontitis. ${ }^{14} \mathrm{We}$ focused on the causal direction from diabetes to periodontal disease in Japanese patients.

\section{Fracture}

Diabetes affects bone metabolism and structure, making it a potential risk factor for fracture and osteoporosis. It has been recognized that oxidative stress and advanced glycation end products caused by hyperglycemia play important roles in deteriorating bone quality. ${ }^{15}$ According to meta-analyses of studies from Western and East Asian countries, the association between diabetes and hip fracture was stronger among patients with type 1 diabetes (relative risk [RR], 6.3-6.9) than among those with type 2 diabetes (RR, 1.4-1.7). ${ }^{16,17}$ A discrepancy in the effect of diabetes on bone mineral density (BMD) between type 1 and type 2 diabetes has also been reported, with evidence that spine and hip BMD decreased among patients with type 1 diabetes but increased among those with type 2 diabetes. ${ }^{16}$ Regardless of $\mathrm{BMD}$, increased fragility of bone structure increases the risk of fracture in patients with diabetes. We focused on the incidence and prevalence of fracture among patients with diabetes in Japan.

\section{Impaired cognitive function and dementia}

The relationship between diabetes and major types of dementia has been discussed in epidemiologic studies and pathophysiological studies. ${ }^{18}$ Diabetes and its associated cardiovascular risk factors result in an increased risk of atherosclerosis and stroke, both of which can cause vessel degeneration in the brain. Hyperglycemia-induced glucose toxicity causes microvascular abnormalities and brain aging. Furthermore, diabetes and its treatment may induce abnormal amyloid metabolism, which can cause Alzheimer-type pathology. ${ }^{18}$ In a recent study in mainly non-Japanese subjects, patients with type 2 diabetes were shown to be at a significantly higher risk of dementia. In that study, the multiple-adjusted pooled RR for any form of dementia being associated with diabetes was 1.73 in men and 1.62 in women. ${ }^{19}$ In this study, we review the available literature on the incidence and prevalence of impaired cognitive function and dementia among patients with diabetes in Japan.

\section{Depression}

Depression is common in patients with diabetes. An international meta-analysis has shown that the odds of having depression 
among patients with diabetes are double those of people without diabetes. ${ }^{20}$ The relationship between diabetes and depression is thought to be bidirectional: depression is a risk factor for developing diabetes, but diabetes is also a risk factor for developing and exacerbating depression. A possible pathophysiological explanation for the causal link between depression and diabetes is dysregulation of key regulatory systems, including the hypothalamic-pituitary-adrenal axis and the sympathoadrenal system, and the increased production of proinflammatory cytokines associated with insulin resistance. ${ }^{21}$ By contrast, diabetes and its complications are also implicated as risk factors for depression, ${ }^{22}$ presumably because of the stress caused by the diagnosis or complications of diabetes and the side effects of antidiabetic drugs. ${ }^{23}$ Based on pooled data of 48,808 patients with diabetes, the presence of type 2 diabetes was associated with a $24 \%$ increased risk of having depression ${ }^{24}$; however, the mechanisms underlying this remain unclear. In this literature review, we focused on diabetes as a risk factor for depression, excluding studies investigating the causal link from depression to developing or aggravating diabetes.

\section{MATERIAL AND METHODS}

\section{Search strategy}

We followed the PRISMA Statement (please see eMaterial 1) ${ }^{25}$; this study has not been registered on the PROSPERO database. We conducted a literature search of the MEDLINE (PubMed) and Ichushi-Web databases (a Japanese database for literature search) for studies published until December 31, 2016. PubMed searches were also conducted using medical subject headings terms. For a start, we searched the literature by including all of the following four words or phrases: "diabetes," "prevalence or incidence," "Japan," and the name of the comorbid condition of interest (eg, "cancer"). If the number of identified studies was fewer than 50 using this method, we repeated the search excluding "prevalence" or "incidence" from the list of search terms. Regarding fracture, we found that including the term "fracture" did not capture the required studies well, and these studies were detected by adding the word "osteoporosis" instead; therefore, we added the term "osteoporosis" to increase coverage. Other search terms used in PubMed are detailed in eTable 1.

For the systematic review, literature was selected in three steps. First, we screened article titles. Second, we checked the content of the abstracts to judge their relevance. Third, we selected articles by reading the whole text of suitable articles. At each step, two authors (HT and NIS) independently extracted the literature, and any disagreement regarding selection was resolved via consensus among all authors. Additional articles were identified using citation tracing in the included articles.

\section{Study selection and evidence classification}

In the current review, we focused on epidemiological studies comparing the incidence and prevalence of the conditions of interest in the general population of Japan. We excluded studies not conducted in Japanese populations and review articles that did not include a meta-analysis. Main measures were set as hazard ratio (HR), incidence rate ratio (IRR), and odds ratios (ORs). The term "relative risk (RR)" used in each reviewed study was carefully converted to one of the following: HR, IRR, and OR, in order to avoid ambiguity. Studies that used disease-specific mortality to confirm disease were excluded because the estimated
Table 1. Evidence classification

\begin{tabular}{lll}
\hline I & $\begin{array}{l}\text { Integrated } \\
\text { analyses }\end{array}$ & Any meta-analyses of cohort and case-control studies \\
II & $\begin{array}{l}\text { Cohort study } \\
\text { design }\end{array}$ & $\begin{array}{l}\text { Comparison of the incidence of a given comorbid } \\
\text { condition between patients with and without diabetes. } \\
\text { The incidence rate ratio of the condition is estimated } \\
\text { or can be estimated regarding diabetes exposure. } \\
\text { There is no distinction between prospective and } \\
\text { retrospective studies. }\end{array}$ \\
III $\begin{array}{l}\text { Case-control } \\
\text { study design } \\
\text { (longitudinal) }\end{array}$ & $\begin{array}{l}\text { Comparison of the proportion with a history of } \\
\text { diabetes among those who did and did not experience } \\
\text { a given comorbid condition. The odds ratio of the } \\
\text { condition is estimated regarding diabetes exposure. } \\
\text { The timing of exposure (diabetes) and the outcome } \\
\text { measurement should be separate (longitudinal). }\end{array}$ \\
IV $\begin{array}{l}\text { Cross-sectional } \\
\text { study design }\end{array}$ & $\begin{array}{l}\text { Comparison of the prevalence of a given comorbid } \\
\text { condition between patients with and without diabetes. } \\
\text { The prevalence ratio or odds ratio is either estimated } \\
\text { or can be estimated. }\end{array}$ \\
\hline
\end{tabular}

RR would be influenced by the different incidence rates for developing the comorbid disease and by the different mortality rates among patients developing that disease. We focused solely on the causal direction from diabetes to common comorbidities, so we excluded longitudinal studies that intended to reveal the effect of those comorbidities on developing diabetes.

For the cross-sectional analyses, we included not only typical cross-sectional studies that investigated the relationship between diabetes and the target disease but also the baseline characteristics in tables of study participants in other studies, regardless of the research questions but provided the study setting did not deviate too far from the general population and provided the crosssectional relationship between diabetes and the disease could be investigated using prevalence ORs.

After screening the identified literature, we classified epidemiologic studies into the following categories: metaanalyses/systematic reviews (ie, integrated analyses), cohort studies, case-control studies (longitudinal), and cross-sectional studies (Table 1). We excluded single-arm cohort studies that included only patients with diabetes and retrospective cohort studies that examined the prevalence of diabetes in only "cases" (ie, those who suffered from comorbid conditions). This was because comparison between the groups within a study would probably have ensured some degree of comparability. Moreover, regardless of whether a study was called a case-control study by its authors, we classified it as a cross-sectional study if the exposure and outcome were measured simultaneously. Regarding cohort and case-control studies, we evaluated the quality using the Newcastle-Ottawa scale (eTable 2 and eTable 3$){ }^{26}$ In this study, we did not conduct a meta-analysis because we found few epidemiological studies regarding diabetes-related conditions in the Japanese population.

\section{RESULTS}

\section{Cancer and diabetes}

We reviewed 67 titles and abstracts of studies looking at the relationship between cancer and diabetes. Among them, we identified three integrated analyses, ${ }^{27-29}$ eight cohort studies, ${ }^{30-37}$ 
Table 2. Number of studies identified through literature search

\begin{tabular}{llccc}
\hline \multicolumn{1}{c}{ Evidence classification $^{\mathrm{a}}$} & I & II & III & IV \\
\hline Cancer & 3 & 8 & 5 & 1 \\
Periodontal disease & 0 & 1 & 0 & 4 \\
Fracture & 0 & 1 & 0 & 4 \\
Impaired cognitive function & 0 & 1 & 0 & 3 \\
Depression & 0 & 0 & 1 & 1 \\
\hline
\end{tabular}

${ }^{\text {a}}$ I: Integrated analyses, II: Cohort study design, III: Case-control study design (longitudinal), IV: Cross-sectional study design.

five case-control studies, ${ }^{38-42}$ and one cross-sectional study (Table 2). ${ }^{43}$

A pooled analysis of eight cohort studies showed that diabetes was associated with statistically significant increased risks of all cancers (HR 1.19; 95\% confidence interval [CI], 1.12-1.25), colon cancer (HR 1.40; 95\% CI, 1.19-1.64), liver cancer (HR 1.97; 95\% CI, 1.65-2.36), pancreatic cancer (HR 1.85; 95\% CI, 1.46-2.34), and bile duct cancer (HR 1.66; 95\% CI, 1.14-2.41; men only). ${ }^{27}$ Another meta-analysis showed that the risk for all cancers was increased in men and women (OR 1.70; 95\% CI, 1.38-2.10), though some of the studies only ascertained the incidence of cancer from death certificates. However, in a sex-specific analysis, diabetes was associated with a significantly increased risk of all cancers in men (adjusted RR 1.25; 95\% CI, 1.06-1.46) and a borderline risk increase in women (adjusted RR 1.23; 95\% CI, 0.97-1.56) based on three cohort studies and one case-control study. ${ }^{28}$ As for site-specific cancer risk, a systematic review was reported of liver cancer in diabetes, and it showed that the IRRs of diabetes for liver cancer were 2.10 (95\% CI, 1.60-2.76) in cohort studies and 2.32 (95\% CI, 1.73-3.12) in case-control studies. ${ }^{29}$

Among eight cohort studies, four analyzed all cancer risk and four analyzed site-specific cancer risk. ${ }^{30-33}$ A representative example of a study examining cancer risk is the Japan Public Health Center-Based Prospective study (JPHC study). In that study, 97,771 community-dwelling Japanese people aged 40-69 years were followed up for 13 years, and there was a $27 \%$ increased risk of total cancer observed in men among those with a history of diabetes compared with those without $(n=3,907$ [366 with diabetes]; HR 1.27; 95\% CI, 1.14-1.42). ${ }^{30}$ Specific cohort studies have reported site-specific cancer risks, including increased risks of pancreatic, ${ }^{34}$ gastric, ${ }^{35,36}$ and prostate ${ }^{37}$ cancer, in patients with diabetes. Case-control studies have also reported increased ORs of all, ${ }^{38}$ hepatocellular, ${ }^{39,40}$ pancreatic, ${ }^{41}$ and ovarian cancer. ${ }^{42}$ In a study reported as being case-control, but in which the exposure and outcome were measured simultaneously (so it was treated as a cross-sectional analysis in this study), there was an elevated OR for uterine endometrial cancer among patients with diabetes (Table 3 ). ${ }^{43}$

\section{Periodontal disease and diabetes}

Eleven studies on the subject of periodontal disease in diabetes were identified through title and abstract review (nine from PubMed and two from additional searches) and were reviewed in detail. After evaluating entire texts, we found one cohort study and four cross-sectional studies, including one study conducted as a case-control study but in which diabetes and periodontal disease status were measured at the same time. We found no integrated analyses (Table 2).

Morita et al reported a cohort study of 5,856 participants (workers in and around Nagoya City) who were followed up for
5 years, focusing on 150 patients (2.6\%) with $\mathrm{HbA} 1 \mathrm{c} \geq 6.5 \%$ at baseline. ${ }^{44}$ The RR for developing periodontal pockets (a Community Periodontal Index of 3 or 4 ) was 1.17 (95\% CI, 1.01-1.36) in those with $\mathrm{HbA1c} \geq 6.5 \%$ at baseline, after adjustment for body mass index, smoking status, sex, and age. ${ }^{44}$ In another study, Marugame et al evaluated alveolar bone loss and showed an adjusted OR of 2.55 (95\% CI, 0.86-7.54) for the complication among 664 Japanese men aged 46-57 years with newly diagnosed type 2 diabetes. ${ }^{45}$

Three studies ${ }^{46-48}$ used secondary data analyses from the same prospective cohort (the Hisayama study). The Hisayama study comprised a series of prospective studies of cerebrovascularcardiovascular disease initiated in 1961 in Hisayama, a suburban town in the Fukuoka metropolitan area of Kyushu Island in Japan. Two studies indicated that there were significant associations between the prevalence of periodontal diseases and impaired fasting glucose (IFG), ${ }^{46}$ impaired glucose tolerance (IGT), and diabetes in women. ${ }^{47}$ Moreover, Saito et al showed that periodontal disease is associated with the development of glucose intolerance. ${ }^{48}$ Despite using data from prospective cohort studies, the estimates derived for the present study were obtained from cross-sectional investigations (Table 3 ). ${ }^{46-48}$

\section{Fractures and diabetes}

We reviewed 21 titles and abstracts concerning the relationship between diabetes and fractures. This revealed one cohort study, four cross-sectional studies, and no integrated analyses (Table 2).

In a hospital-based cohort study from the Nagano Cohort, the authors reported the follow-up data for 1,614 postmenopausal women $(8.1 \%$ had diabetes) over a median period of 6.7 years to evaluate the association between lifestyle-related diseases and fractures. The ORs for fracture among patients with diabetes compared with those without diabetes were 2.33 (95\% CI, 0.96-5.65) for femoral neck fracture, 0.80 (95\% CI, 0.49-1.29) for vertebral fracture, and 1.20 (95\% CI, 0.66-2.19) for long bone fracture. ${ }^{49}$

A population-based, multicenter, cross-sectional survey investigating postmenopausal osteoporosis in Chiba City was reported for the period from 2001 to 2009 (the Chiba Bone Survey). ${ }^{50}$ Among the 64,809 women aged $>40$ years, the authors identified that 2,116 had diabetes and that osteoporosis (BMD $<74 \%$ of young adult mean) was present in $22.5 \%$ of patients with diabetes and $15.6 \%$ of individuals without diabetes. Fracture during the most recent 5 years occurred in $7.6 \%$ of patients with diabetes, which was significantly higher than the rate of $5.2 \%$ that occurred in patients without diabetes $(P<0.001)$. The adjusted OR for bone fracture during the most recent 5 years was 1.26 (95\% CI, $1.05-1.50)$ in patients with diabetes.

There were also three hospital-based studies conducted by the Shimane University group, but it should be noted that there may have been an overlap of the study participants. ${ }^{51-53}$ In one of these studies, 298 patients with type 2 diabetes were compared with nondiabetic controls. ${ }^{52}$ Among patients with type 2 diabetes, 43 women $(31.4 \%)$ and 61 men $(37.9 \%)$ had vertebral fractures, whereas in the control group, the percentages were $24.9 \%$ and $14.5 \%$, respectively. Logistic regression analysis showed that type 2 diabetes was an independent risk factor for the prevalence of vertebral fracture in women (OR 1.86; 95\% CI, 1.11-3.12) and men (OR 4.73; 95\% CI, 2.19-10.20) after adjustment for age, body mass index, and BMD of the lumber spine (Table 3). ${ }^{52}$ 
Tanaka H, et al.

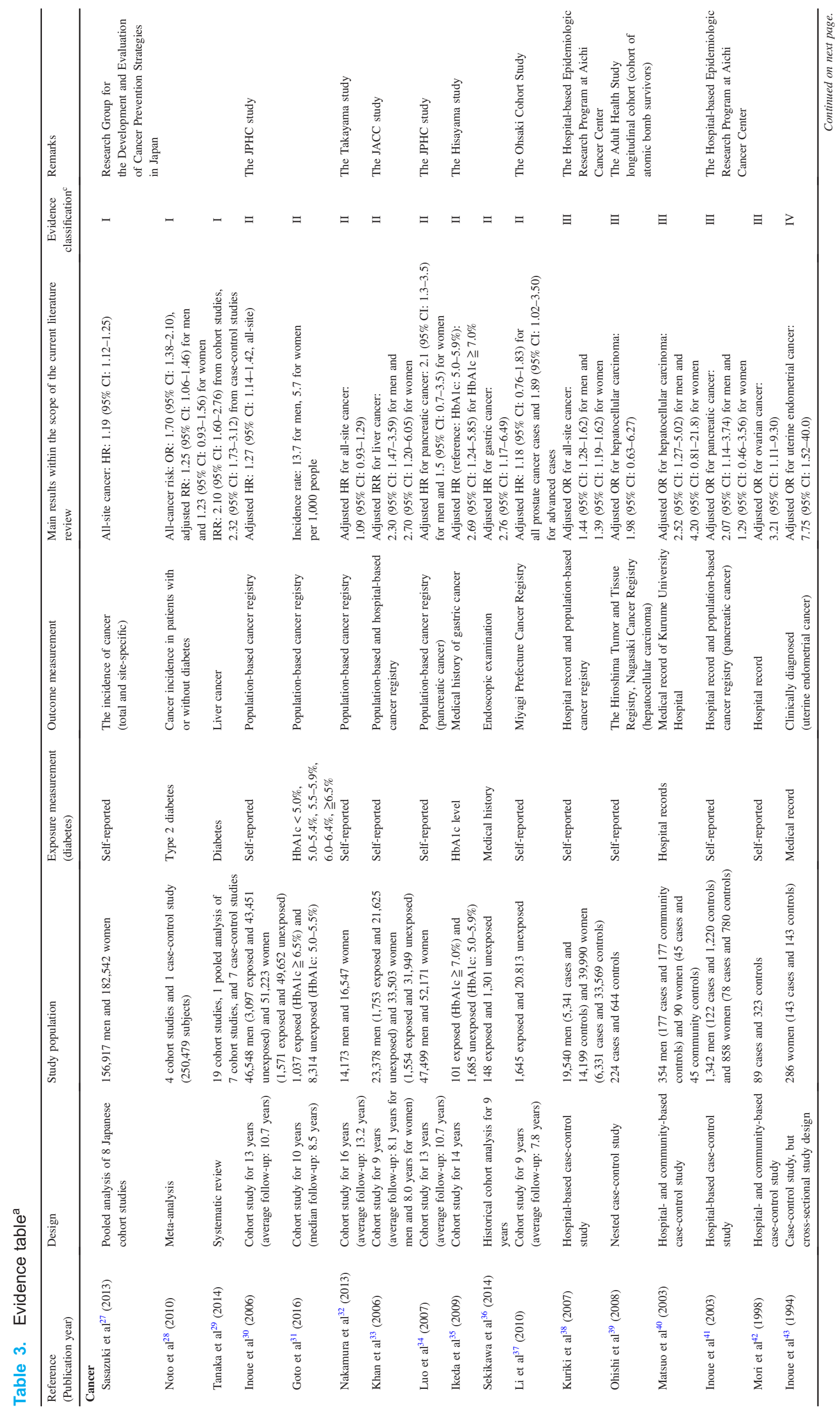


Diabetes and Incidence/Prevalence of Comorbidities in Japan

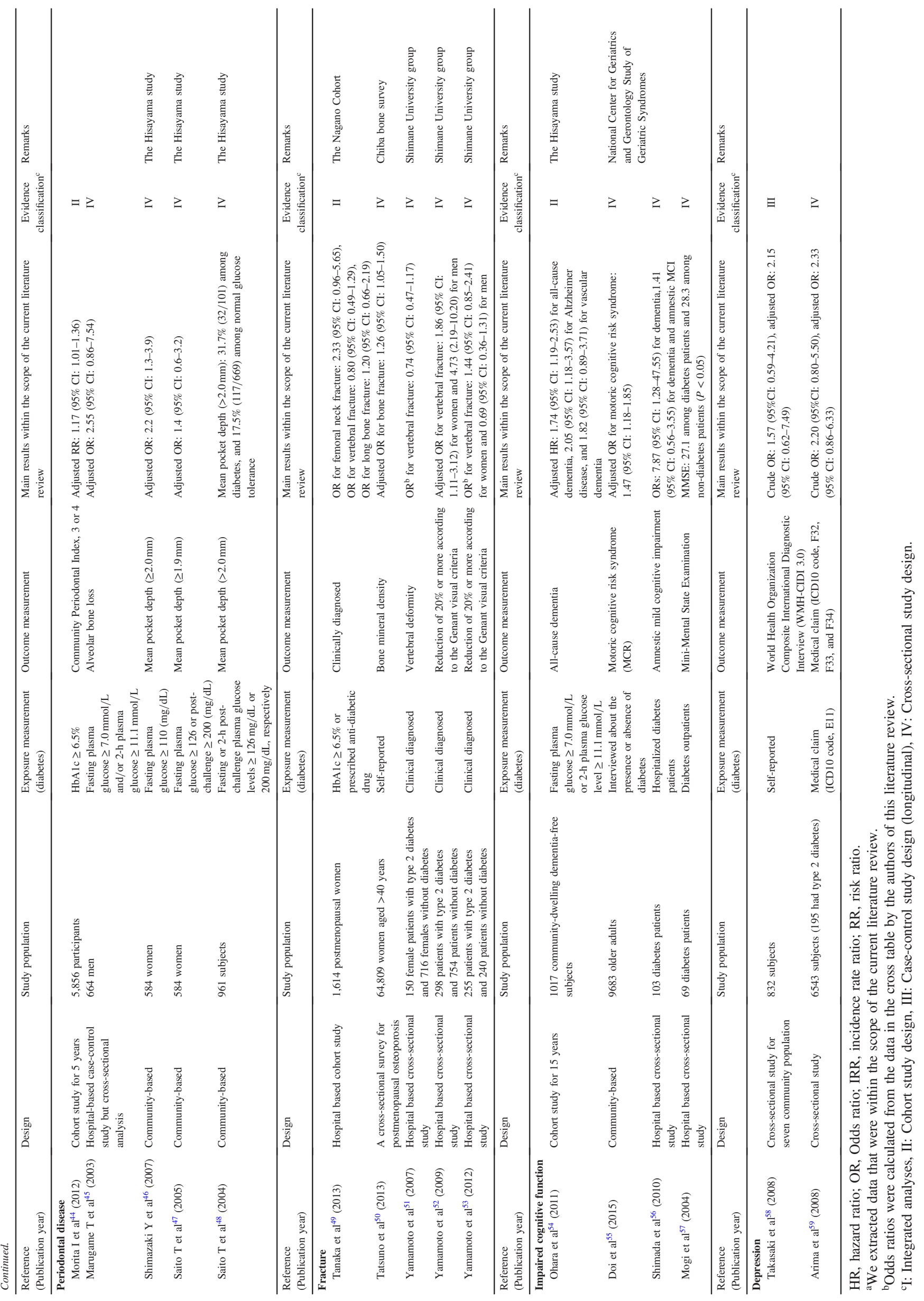




\section{Impaired cognitive function and diabetes}

We reviewed 14 titles and abstracts reporting the association between diabetes and impaired cognitive function, including mild cognitive impairment (MCI) in Japanese populations. This revealed one cohort study and three cross-sectional studies (Table 2).

Population-based evidence was reported from the Hisayama study. ${ }^{54}$ In the study, community-dwelling subjects with dementia who were aged 60 years or older $(n=1,017)$ were divided into five groups according to the results of a 75-g oral glucose tolerance test (normal glucose tolerance, IFG, IGT, both IFG and IGT, and diabetes). ${ }^{54}$ Over a 15 -year follow-up period, diabetes was shown to be a significant risk factor for the development of all-cause dementia (adjusted HR 1.74; 95\% CI, 1.19-2.53), Alzheimer's disease (adjusted HR 2.05; 95\% CI, 1.18-3.57), and vascular dementia (adjusted HR 1.82; 95\% CI, 0.89-3.71).

A cross-sectional study was carried out by Doi et al to reveal the prevalence and risk factors of motoric cognitive risk (MCR) syndrome. They aimed to report the prevalence of, and modifiable factors associated with, MCR in Japanese community-dwelling adults and screened 9,683 older adults (52\% women, mean age: 73.6 years). Among the patients with MCR, 20.4\% had diabetes, and among the patients without MCR, $12.4 \%$ had diabetes $(P<0.001)$. After adjusting for several covariates, diabetes was associated with an increased risk of MCR (OR 1.47; 95\% CI, $1.18-1.85) .^{55}$

The prevalence of MCI was investigated in another crosssectional study. Among 103 patients with diabetes, 22 (21\%) had dementia and $9(9 \%)$ had amnestic MCI, while in a group of 30 controls, 1 (3\%) had dementia and $6(20 \%)$ had amnestic MCI. The ORs were 7.87 (95\% CI, 1.28-47.55) for dementia and 1.41 (95\% CI, 0.56-3.55) for dementia and amnestic MCI. ${ }^{56}$ The final cross-sectional study investigated cognitive function in Japanese patients with diabetes $(n=69)$ and showed that they had significantly worse Mini-Mental State Examination scores than those without diabetes $(n=27)$ (27.1 and 28.3, respectively; $P<0.05)$ (Table 3). ${ }^{57}$

\section{Depression and diabetes}

We reviewed 18 titles and abstracts for studies reporting the association between diabetes and depression. Among these, there was one case-control study and one cross-sectional study that met our inclusion criteria (Table 2).

Takasaki et al reported a community-based case-control study (World Mental Health Japan, 2002-2004) of two urban and five rural community areas in which they examined the association between depression and circulatory diseases, including diabetes. ${ }^{58}$ They used the World Health Organization Composite International Diagnostic Interview Version 3.0 to assess major depression. The reported crude OR was 1.57 (95\% CI, 0.59-4.21), with diabetes being associated with 2.15-fold higher odds of developing major depression after adjusting for sex, age, smoking status, alcohol intake, and education; however, the association was not statistically significant (95\% CI, 0.62-7.49). In the cross-sectional study, claims data and a self-reported questionnaire were used for 6,543 Japanese employees aged 18-65 years (195 with type 2 diabetes and 6,348 without type 2 diabetes). ${ }^{59}$ The prevalence of comorbid depression was $2.6 \%$ in the group with diabetes but only $1.2 \%$ in the group without diabetes (crude OR 2.20; 95\% CI, 0.88-5.50). After adjustment for age, gender, alcohol intake, smoking status, exercise, and dietary restriction, the OR for depression in type 2 diabetes was 2.33 (95\% CI, 0.86-6.33) (Table 3).

\section{DISCUSSION}

\section{Main findings}

We reviewed studies in Japan that examined comorbid conditions recently linked to diabetes. Although there were several cohort studies and meta-analyses regarding the relationship for cancer in diabetes, there was scant epidemiological evidence for the other four conditions. Indeed, only one cohort study each was identified for periodontal disease, fracture, and cognitive impairment. The remaining evidence was either cross-sectional or collected from tables of baseline characteristics in studies with other aims. However, some cohort studies had notable limitations, particularly in terms of the study setting and sample size. Regarding the evaluation of the Newcastle-Ottawa scale, the scores were high in both cohort and case-control studies; however, we found it difficult to judge the overall tendency due to the inadequate number of studies. Overall, our results underscore the need for more epidemiological evidence about these newly recognized comorbidities in Japan. Only then can we gain valid insights to help us plan effective management of these conditions. By conducting this systematic review of epidemiological studies, we have highlighted the areas where the best research has been done, thereby highlighting which conditions future epidemiological studies should target. We believe that further epidemiological studies will help to obtain detailed information on the influence of diabetes on comorbidities, which would contribute to forthcoming revision of guidelines for diabetes care in Japan.

\section{Interpretation}

For cancer, we found epidemiological studies of relatively high quality investigating the relationship with diabetes. Several studies, such as the JPHC study, were community-based and well designed and had good generalizability to the general Japanese population. However, some research questions remain unresolved. For example, diabetes in general has been reported to be protective for prostate cancer in many non-Japanese populations, ${ }^{60}$ but we found the opposite effect in the Japanese population. ${ }^{37}$ The reason for this discrepancy may be that the impact of diabetes on cancer is different in Asian and western populations. Further research in Japanese populations would help clarify this relationship, and there are several ongoing prospective cohort studies in Japan. ${ }^{61-63}$ Further observation and analysis should provide clarifying epidemiological evidence.

In contrast to the range of studies for cancer, there were quite limited numbers of studies for the other four conditions. The Hisayama study, a population-based prospective cohort study, was notable among these, demonstrating that a history of diabetes was a significant risk factor for the development of all-cause dementia, Alzheimer's disease, and vascular dementia. A part of the Hisayama study was included in a pooled international analysis that showed that diabetes patients have approximately 60-70\% greater risk for the development of dementia compared with those without diabetes. ${ }^{19}$ However, there was a paucity of good-quality cohort studies for periodontal disease and fracture, with some being old and/or having small sample sizes and others being hospital-based and lacking generalizability. For example, although Morita et al tried to investigate the relationship between diabetes and periodontal disease, their results should be 
interpreted with caution in terms of external validity because the cohort was derived from employed workers receiving annual medical checkups. By contrast, a population-based cohort study of 2,626 community-dwelling Germans in 2012 substantially advanced previous research on the influence of etiology and glycemic control on periodontal disease, using population-based longitudinal data. ${ }^{64}$ Therefore, we concluded that the studies investigating the relationship between diabetes and periodontal disease, fracture, and depression in Japan have been too few to compare with studies in other countries. Well-designed prospective cohort studies can help understand the newly recognized diabetes-related conditions among patients in Japan.

Some efforts could increase the quality and quantity of epidemiological evidence in this field. First, when researchers investigate the relationship between diabetes as an exposure and the complications as the outcome in cohort studies, they need to follow the occurrence of these complications in participants. To make it easy to capture the occurrence, the development of valid and feasible measurements is necessary. For example, to follow the occurrence of periodontal diseases, some studies have used the Community Periodontal Index, ${ }^{41}$ whereas others have used alveolar bone loss. ${ }^{45}$ Like these criteria, it is important to give explicit criteria for the diagnosis of complications if we are to ensure optimal intra- and inter-rater reliability that will improve the interpretability of results. Moreover, establishing common criteria will facilitate the implementation of epidemiological studies by rendering study design easier and between-study comparison more straightforward.

Second, utilizing existing cohorts will lower the cost and time needed to obtain new evidence. Some complications may also be too expensive to follow up for all the cohort members; in this case, researchers may want to use more efficient study designs, such as a case-cohort design.

Third, utilization of existing data (eg, claims data) may be another efficient way to obtain clinical data, although it has inherent limitations. Antidiabetic medicine use can be utilized to detect individuals with diabetes with high specificity; however, it can misclassify patients not using medication. In addition, detecting periodontal disease and dementia may be almost impossible.

Given the limitations of current epidemiological studies, combining insights gained from several types of studies is necessary to grasp the full landscape of diabetes and its complications. Furthermore, the strategic allocation of research grants in this field is needed to conduct more studies of higher quality.

\section{Limitations}

There are several limitations in this study. First, although we attempted to include all studies of interest by conducting an extensive systematic review, we may have failed to include a few studies, especially those with cross-sectional designs. This is because some of our cross-sectional evidence came from the tables of baseline characteristics in studies done for other reasons. Second, we did not include evidence from single-arm studies, such as those that estimated the incidence of comorbid conditions among patients with diabetes only. We decided not to include these studies because they may lack comparability with other studies. If comparability could be ensured, combining single-arm studies could be an efficient strategy for estimating incidence rates. For example, the Food and Drug Administration allows registry data to be used to provide historical controls to replace the post-approval study requirement for pharmaceutical approval reviews. ${ }^{65}$ Combining a single-arm clinical trial with historical controls from existing databases during the pharmaceutical application process is analogous to combining a single-arm epidemiological cohort of patients with diabetes and a community-based cohort that is not restricted to patients with diabetes. This could generate epidemiological findings about the incidence of comorbid conditions among patients with diabetes compared with the general population. Third, evidence of inverse causal relationships (ie, from comorbid conditions to diabetes) was beyond the scope of this study. Because it is highly likely that the five comorbidities we dealt with could lead to the development of diabetes or the worsening of glycemic control, further review might be needed to illuminate the whole relationship between diabetes and its comorbidities.

\section{Conclusion}

In Japan, there is scant evidence about the effect of diabetes on the incidence and prevalence of periodontal disease, fracture, impaired cognitive function and dementia, and depression. By contrast, there is much stronger evidence for the relationship of diabetes with cancer. Future efforts are needed to improve the quality and quantity of evidence specific to the Japanese population if we are to provide evidence-based interventions against these putative complications of diabetes.

\section{ACKNOWLEDGEMENTS}

This study was supported by grants from the National Center for Global Health and Medicine (26-s002).

Conflicts of interest: None declared.

\section{APPENDIX A. SUPPLEMENTARY DATA}

Supplementary data related to this article can be found at https:// doi.org/10.2188/jea.JE20170155.

\section{REFERENCES}

1. Laiteerapong N, Karter AJ, Liu JY, et al. Correlates of quality of life in older adults with diabetes: the diabetes \& aging study. Diabetes Care. 2011;34(8):1749-1753.

2. American Diabetes Association. Standards of medical care in diabetes. Diabetes Care. 2017;40(Suppl 1):S1-S135.

3. Global Burden of Metabolic Risk Factors for Chronic Diseases Collaboration. Cardiovascular disease, chronic kidney disease, and diabetes mortality burden of cardiometabolic risk factors from 1980 to 2010: a comparative risk assessment. Lancet Diabetes Endocrinol. 2014;2(8):634-647.

4. Ogurtsova K, da Rocha Fernandes JD, Huang Y, et al. IDF Diabetes Atlas: global estimates for the prevalence of diabetes for 2015 and 2040. Diabetes Res Clin Pract. 2017;128:40-50.

5. NCD Risk Factor Collaboration (NCD-RisC). Worldwide trends in diabetes since 1980: a pooled analysis of 751 population-based studies with 4.4 million participants. Lancet. 2016;387(10027): 1513-1530.

6. Bardenheier BH, Lin J, Zhuo X, et al. Disability-free life-years lost among adults aged $\geq 50$ years with and without diabetes. Diabetes Care. 2016;39(7):1222-1229.

7. Tajima N, Noda M, Origasa H, et al. Evidence-based practice guideline for the treatment for diabetes in Japan 2013. Diabetol Int. 2015;6:151-187.

8. Giovannucci E, Harlan DM, Archer MC, et al. Diabetes and cancer: 
a consensus report. Diabetes Care. 2010;33(7):1674-1685.

9. Kasuga M, Ueki K, Tajima N, et al. Report of the Japan Diabetes Society/Japanese Cancer Association Joint Committee on Diabetes and Cancer. Cancer Sci. 2013;104(7):965-976.

10. Tsilidis KK, Kasimis JC, Lopez DS, Ntzani EE, Ioannidis JP. Type 2 diabetes and cancer: umbrella review of meta-analyses of observational studies. BMJ. 2015;350:g7607.

11. Nelson RG, Shlossman M, Budding LM, et al. Periodontal disease and NIDDM in Pima Indians. Diabetes Care. 1990;13(8):836-840.

12. Tsai C, Hayes C, Taylor GW. Glycemic control of type 2 diabetes and severe periodontal disease in the US adult population. Community Dent Oral Epidemiol. 2002;30(3):182-192.

13. Demmer RT, Squillaro A, Papapanou PN, et al. Periodontal infection, systemic inflammation, and insulin resistance: results from the continuous National Health and Nutrition Examination Survey (NHANES) 1999-2004. Diabetes Care. 2012;35(11):22352242.

14. Preshaw PM, Alba AL, Herrera D, et al. Periodontitis and diabetes: a two-way relationship. Diabetologia. 2012;55(1):21-31.

15. Saito M, Fujii K, Mori Y, Marumo K. Role of collagen enzymatic and glycation induced cross-links as a determinant of bone quality in spontaneously diabetic WBN/Kob rats. Osteoporos Int. 2006; 17(10):1514-1523.

16. Vestergaard P. Discrepancies in bone mineral density and fracture risk in patients with type 1 and type 2 diabetes-a meta-analysis. Osteoporos Int. 2007;18(4):427-444.

17. Janghorbani M, Van Dam RM, Willett WC, Hu FB. Systematic review of type 1 and type 2 diabetes mellitus and risk of fracture. Am J Epidemiol. 2007;166(5):495-505.

18. Biessels GJ, Staekenborg S, Brunner E, Brayne C, Scheltens P. Risk of dementia in diabetes mellitus: a systematic review. Lancet Neurol. 2006;5(1):64-74.

19. Chatterjee S, Peters SA, Woodward M, et al. Type 2 diabetes as a risk factor for dementia in women compared with men: a pooled analysis of 2.3 million people comprising more than 100,000 cases of dementia. Diabetes Care. 2016;39(2):300-307.

20. Anderson RJ, Freedland KE, Clouse RE, Lustman PJ. The prevalence of comorbid depression in adults with diabetes: a metaanalysis. Diabetes Care. 2001;24(6):1069-1078.

21. Golden SH. A review of the evidence for a neuroendocrine link between stress, depression and diabetes mellitus. Curr Diabetes Rev. 2007;3(4):252-259.

22. de Groot M, Anderson R, Freedland KE, Clouse RE, Lustman PJ. Association of depression and diabetes complications: a metaanalysis. Psychosom Med. 2001;63(4):619-630.

23. Tabák AG, Akbaraly TN, Batty GD, Kivimäki M. Depression and type 2 diabetes: a causal association? Lancet Diabetes Endocrinol. 2014;2(3):236-245

24. Nouwen A, Winkley K, Twisk J, et al; European Depression in Diabetes (EDID) Research Consortium. Type 2 diabetes mellitus as a risk factor for the onset of depression: a systematic review and meta-analysis. Diabetologia. 2010;53(12):2480-2486.

25. Moher D, Liberati A, Tetzlaff J, Altman DG; PRISMA Group. Preferred reporting items for systematic reviews and meta-analyses: the PRISMA statement. PLoS Med. 2009;6:e1000097.

26. Wells G, Shea B, O'Connell D, et al. The Newcastle-Ottawa Scale (NOS) for assessing the quality of nonrandomised studies in meta-analyses. Ottawa, Ontario, Canada: Ottawa Hospital Research Institute; 2013. www.ohri.ca/programs/clinical_epidemiology/ oxford.asp [accessed on 18 October 2017].

27. Sasazuki S, Charvat H, Hara A, et al; Research Group for the Development and Evaluation of Cancer Prevention Strategies in Japan. Diabetes mellitus and cancer risk: pooled analysis of eight cohort studies in Japan. Cancer Sci. 2013;104(11):1499-1507.

28. Noto H, Osame K, Sasazuki T, Noda M. Substantially increased risk of cancer in patients with diabetes mellitus: a systematic review and meta-analysis of epidemiologic evidence in Japan. $J$ Diabetes Complications. 2010;24(5):345-353.

29. Tanaka K, Tsuji I, Tamakoshi A, et al; Research Group for the Development and Evaluation of Cancer Prevention Strategies in
Japan. Diabetes mellitus and liver cancer risk: an evaluation based on a systematic review of epidemiologic evidence among the Japanese population. Jpn J Clin Oncol. 2014;44(10):986-999.

30. Inoue M, Iwasaki M, Otani T, Sasazuki S, Noda M, Tsugane S. Diabetes mellitus and the risk of cancer: results from a large-scale population-based cohort study in Japan. Arch Intern Med. 2006; 166(17):1871-1877.

31. Goto A, Noda M, Sawada N, et al; JPHC Study Group. High hemoglobin A1c levels within the non-diabetic range are associated with the risk of all cancers. Int J Cancer. 2016;138(7):1741-1753.

32. Nakamura K, Wada K, Tamai Y, et al. Diabetes mellitus and risk of cancer in Takayama: a population-based prospective cohort study in Japan. Cancer Sci. 2013;104(10):1362-1367.

33. Khan M, Mori M, Fujino Y, et al; Japan Collaborative Cohort Study Group. Site-specific cancer risk due to diabetes mellitus history: evidence from the Japan Collaborative Cohort (JACC) Study. Asian Pac J Cancer Prev. 2006;7(2):253-259.

34. Luo J, Iwasaki M, Inoue M, et al; JPHC Study Group. Body mass index, physical activity and the risk of pancreatic cancer in relation to smoking status and history of diabetes: a large-scale populationbased cohort study in Japan-the JPHC study. Cancer Causes Control. 2007;18(6):603-612.

35. Ikeda F, Doi Y, Yonemoto K, et al. Hyperglycemia increases risk of gastric cancer posed by Helicobacter pylori infection: a populationbased cohort study. Gastroenterology. 2009;136(4):1234-1241.

36. Sekikawa A, Fukui H, Maruo T, Tsumura T, Okabe Y, Osaki Y. Diabetes mellitus increases the risk of early gastric cancer development. Eur J Cancer. 2014;50(12):2065-2071.

37. Li Q, Kuriyama S, Kakizaki M, et al. History of diabetes mellitus and the risk of prostate cancer: the Ohsaki Cohort Study. Cancer Causes Control. 2010;21(7):1025-1032.

38. Kuriki K, Hirose K, Tajima K. Diabetes and cancer risk for all and specific sites among Japanese men and women. Eur J Cancer Prev. 2007;16(1):83-89.

39. Ohishi W, Fujiwara S, Cologne JB, et al. Risk factors for hepatocellular carcinoma in a Japanese population: a nested casecontrol study. Cancer Epidemiol Biomarkers Prev. 2008;17(4):846854.

40. Matsuo M. Association between diabetes mellitus and hepatocellular carcinoma: results of a hospital- and community-based case-control study. Kurume Med J. 2003;50(3-4):91-98.

41. Inoue M, Tajima K, Takezaki T, et al. Epidemiology of pancreatic cancer in Japan: a nested case-control study from the Hospitalbased Epidemiologic Research Program at Aichi Cancer Center (HERPACC). Int J Epidemiol. 2003;32(2):257-262.

42. Mori M, Nishida T, Sugiyama T, et al. Anthropometric and other risk factors for ovarian cancer in a case-control study. Jpn J Cancer Res. 1998;89(3):246-253.

43. Inoue M, Okayama A, Fujita M, Enomoto T, Tanizawa O, Ueshima H. A case-control study on risk factors for uterine endometrial cancer in Japan. Jpn J Cancer Res. 1994;85(4):346-350.

44. Morita I, Inagaki K, Nakamura F, et al. Relationship between periodontal status and levels of glycated hemoglobin. J Dent Res. 2012;91(2):161-166

45. Marugame T, Hayasaki H, Lee K, Eguchi H, Matsumoto S. Alveolar bone loss associated with glucose tolerance in Japanese men. Diabet Med. 2003;20(9):746-751.

46. Shimazaki Y, Saito T, Yonemoto K, Kiyohara Y, Iida M, Yamashita Y. Relationship of metabolic syndrome to periodontal disease in Japanese women: the Hisayama Study. J Dent Res. 2007;86(3):271275 .

47. Saito T, Shimazaki Y, Kiyohara Y, et al. Relationship between obesity, glucose tolerance, and periodontal disease in Japanese women: the Hisayama study. J Periodontal Res. 2005;40(4):346353.

48. Saito T, Shimazaki Y, Kiyohara Y, et al. The severity of periodontal disease is associated with the development of glucose intolerance in non-diabetics: the Hisayama study. J Dent Res. 2004;83(6):485-490.

49. Tanaka S, Kuroda T, Saito M, et al. Improvement of fracture risk assessment tool by lifestyle-related diseases. Osteoporosis Japan. 
2013;21(4):723-726 (in Japanese).

50. Tatsuno I, Terano T, Nakamura M, et al. Lifestyle and osteoporosis in middle-aged and elderly women: Chiba bone survey. Endocr J. 2013;60(5):643-650.

51. Yamamoto M, Yamaguchi T, Yamauchi M, Kaji H, Sugimoto T. Bone mineral density is not sensitive enough to assess the risk of vertebral fractures in type 2 diabetic women. Calcif Tissue Int. 2007;80(6):353-358.

52. Yamamoto M, Yamaguchi T, Yamauchi M, Kaji H, Sugimoto T. Diabetic patients have an increased risk of vertebral fractures independent of BMD or diabetic complications. J Bone Miner Res. 2009;24(4):702-709.

53. Yamamoto M, Yamaguchi T, Nawata K, Yamauchi M, Sugimoto T. Decreased PTH levels accompanied by low bone formation are associated with vertebral fractures in postmenopausal women with type 2 diabetes. J Clin Endocrinol Metab. 2012;97(4):1277-1284.

54. Ohara T, Doi Y, Ninomiya T, et al. Glucose tolerance status and risk of dementia in the community: the Hisayama study. Neurology. 2011;77(12):1126-1134.

55. Doi T, Verghese J, Shimada H, et al. Motoric cognitive risk syndrome: prevalence and risk factors in Japanese seniors. J Am Med Dir Assoc. 2015;16(12):1103.e21-1103.e25.

56. Shimada H, Miki T, Tamura A, Ataka S, Emoto M, Nishizawa Y. Neuropsychological status of elderly patients with diabetes mellitus. Diabetes Res Clin Pract. 2010;87(2):224-227.

57. Mogi N, Umegaki H, Hattori A, et al. Cognitive function in Japanese elderly with type 2 diabetes mellitus. J Diabetes Complications. 2004;18(1):42-46.
58. Takasaki Y, Kawakami N, Tsuchiya M, et al. Heart disease, other circulatory diseases, and onset of major depression among community residents in Japan: results of the World Mental Health Survey Japan 2002-2004. Acta Med Okayama. 2008;62(4):241-249.

59. Arima H, Miwa M, Kawahara K. The prevalence of co-morbid depression among employees with type 2 diabetes in a Japanese corporation: a descriptive study using an integrated health database. J Med Dent Sci. 2007;54(1):39-48.

60. Bansal D, Bhansali A, Kapil G, Undela K, Tiwari P. Type 2 diabetes and risk of prostate cancer: a meta-analysis of observational studies. Prostate Cancer Prostatic Dis. 2013;16(2):151-158, S1.

61. Tsugane S, Sobue T. Baseline survey of JPHC study-design and participation rate. Japan Public Health Center-based Prospective Study on Cancer and Cardiovascular Diseases. J Epidemiol. 2001; 11(6 Suppl):S24-S29.

62. Ohno Y, Tamakoshi A; JACC Study Group. Japan collaborative cohort study for evaluation of cancer risk sponsored by monbusho (JACC study). J Epidemiol. 2001;11(4):144-150.

63. Kuriyama S, Nakaya N, Ohmori-Matsuda K, et al. The Ohsaki Cohort 2006 Study: design of study and profile of participants at baseline. J Epidemiol. 2010;20(3):253-258.

64. Demmer RT, Holtfreter B, Desvarieux M, et al. The influence of type 1 and type 2 diabetes on periodontal disease progression: prospective results from the Study of Health in Pomerania (SHIP). Diabetes Care. 2012;35(10):2036-2042.

65. Faris O, Shuren J. An FDA viewpoint on unique considerations for medical-device clinical trials. N Engl J Med. 2017;376(14):13501357. 